\title{
A Two-Parameter Accelerating FODO Cell
}

\author{
Leo Michelotti \\ Fermilab*, P.O.Box 500, Batavia, IL 60510

\section{TRANSFER MATRIX OF AN ACCELERATING FODO CELL}

Abstract

A beamline containing accelerating cavities sandwiched between focusing and defocusing quadrupoles is made periodic by (a) postulating the simplest effect of cavities on transverse dynamics and (b) adopting a fiction that the ratio of initial to final momenta, $\left(p_{i} / p_{f}\right)$ - not the difference in energies, $\left(E_{f}-E_{i}\right)$ - is the same for all cavities. While not realistic, the optics of such a linac can be expressed without reference to initial beam parameters, using methods devised for storage rings. We will derive results for a simple twoparameter thin lens model, the parameters being $p_{i} / p_{f}$ and the ratio of quadrupole spacing to focal length.

Despite substantive differences, a similar language is used to describe the optics of linacs and storage rings. The periodicity of storage rings allows unambiguous definitions of "global lattice functions" - e.g., dispersion, beta, and phase advance - as properties of the machines themselves, with no reference to beam parameters other than energy. On the other hand, the "beta function" of a linac or the "phase advance" between two points in a linac cannot be defined in the same way; the boundary condition is not periodic but typically refers to a set of initial beam parameters. Further, calculating lattice functions for a storage ring rigorously requires a constant energy machine. This is extended to synchrotrons by assuming that the fields change adiabatically, over many turns. In contrast, acceleration in a linac can take place between consecutive quadrupoles.

Suppose that we apply standard formulae involving lattice functions from the theory of storage rings - which we will call "circular reasoning" (CR) - to a linac - whose theory we will call "straight thinking" (ST). What magnitude of error is made if they are applied without modification? We will address this question in the context of a highly simplified model that straddles the two theories, a periodic structure that is a straightforward generalization of CR's FODO cell: the accelerating FODO cell. It will possess three formal parameters: the length of a drift section, the focal lengths of the quadrupoles, and a number representing the energy gain of the accelerating structure. However, the drift's length serves only to set a scale, so our model will actually possess only two essential parameters.

* Operated by the Universities Research Association, Inc. under contract DE-AC02-76CH00300 with the U.S. Department of Energy.
A simple accelerating FODO cell is sketched in Figure 1. A drift section, not a bending magnet, separates the quads,

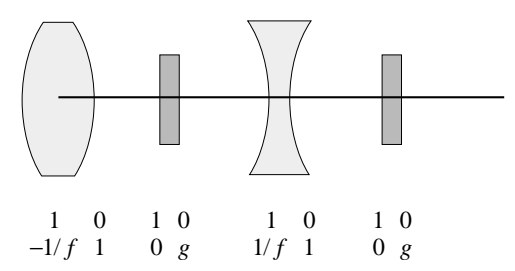

Figure 1: Sketch of an accelerating FODO cell.

and in it is placed a "thin" (zero length) accelerating structure. The quadrupoles are also thin, and their magnetic fields are arranged so that the focal length remains constant; that is, their gradients scale with momentum.

Ignoring edge focusing, the first order effect of the accelerating structure on transvere coordinates is to "rescale" the transverse momentum - or, equivalently, to reduce the transverse velocity - as represented by the transfer matrix,

$$
\left(\begin{array}{c}
x \\
x^{\prime}
\end{array}\right)_{(f)}=\left(\begin{array}{cc}
1 & 0 \\
0 & p_{i} / p_{f}
\end{array}\right) \cdot\left(\begin{array}{c}
x \\
x^{\prime}
\end{array}\right)_{(i)} .
$$

Here $p_{i}$ and $p_{f}$ are the momenta of the particle upon entering and exiting the structure. We define $g \equiv p_{i} / p_{f}$ and write the transfer matrix for the full "drift" section.

$$
\begin{aligned}
\underline{\underline{O}} & =\left(\begin{array}{cc}
1 & l / 2 \\
0 & 1
\end{array}\right)\left(\begin{array}{ll}
1 & 0 \\
0 & g
\end{array}\right)\left(\begin{array}{cc}
1 & l / 2 \\
0 & 1
\end{array}\right) \\
& =\left(\begin{array}{cc}
1 & l(1+g) / 2 \\
0 & g
\end{array}\right)
\end{aligned}
$$

In the limit of no acceleration, $g=1$, while $g=0$ represents extreme acceleration. For highly relativistic particles,

$$
p_{i} / p_{f} \approx E_{i} / E_{f}=\frac{1}{1+\left(E_{f}-E_{i}\right) / E_{i}}=\frac{1}{1+\Delta E / E}
$$

The transfer matrices through the quads are the same as those used in CR, so we can immediately write the full transfer matrix through the accelerating FODO cell, as has been done in Eq.(1). We are finessing the question, "What is $x^{\prime}$ ?" The coordinate that makes a drift a linear element is not the same as the one that makes a thin quadrupole a linear element. The former requires $x^{\prime}=p_{x} / p_{3}=d x / d s$ where $p_{x}$ is the transverse and $p_{3}$ the longitudinal momentum, and $d s$ is longitudinal differential arc length - while 


$$
\begin{aligned}
\underline{\underline{M}} & =\underline{\underline{F}} \cdot \underline{\underline{O}} \cdot \underline{\underline{D}} \cdot \underline{\underline{O}} \\
& =\left(\begin{array}{cc}
1 & 0 \\
-1 / f & 1
\end{array}\right)\left(\begin{array}{cc}
1 & l(1+g) / 2 \\
0 & g
\end{array}\right)\left(\begin{array}{cc}
1 & 0 \\
1 / f & 1
\end{array}\right)\left(\begin{array}{cc}
1 & l(1+g) / 2 \\
0 & g
\end{array}\right) \\
& =\left(\begin{array}{cc}
1+(l / 2 f)(1+g) & (1+g)^{2}(l / 2)(1+l / 2 f) \\
+(1 / f)(g-1-(l / 2 f)(1+g)) & -(l / 2 f)(1+g)+\left(g^{2}-((l / 2 f)(1+g))^{2}\right)
\end{array}\right)
\end{aligned}
$$

the latter uses $x^{\prime}=p_{x} / p$ - where $p$ is the total momentum of the particle, used as a reference scaling parameter. In confusing these two coordinates throughout the calculation, we implicitly require that $p \approx p_{3}$ and insist that the resulting equations are valid only to first order in the transverse coordinates.

\section{EIGENVALUES}

The eigenvalues of $\underline{\underline{M}}$ determine the phase advance and amplitude reduction per cell. Because $\underline{\underline{M}}$ is a matrix of real numbers, its eigenvalues come in complex conjugate pairs; because it is not symplectic, we do not expect the eigenvalues to lie on the unit circle. We express them as $\exp (-\Gamma \pm i \mu)$ and obtain them easily as follows.

$$
\begin{aligned}
\operatorname{det}(\underline{\underline{M}}) & =\left(e^{-\Gamma}\right)^{2}=g^{2} \\
\operatorname{Tr}(\underline{\underline{M}}) & =2 e^{-\Gamma} \cos \mu \\
& =1+g^{2}-\left(\frac{l}{2 f}(1+g)\right)^{2}
\end{aligned}
$$

The first equation is written quickly by noting that the determinant of a product is the product of the determinants and that $\operatorname{det}(\underline{\underline{O}})=g$, while $\operatorname{det}(\underline{\underline{F}})=\operatorname{det}(\underline{\underline{D}})=1$. Rearranging terms slightly provides the results,

$$
\begin{aligned}
g & =e^{-\Gamma} \\
\left(\frac{l}{2 f}\right)^{2} & =\frac{1+g^{2}-2 g \cos \mu}{(1+g)^{2}} \\
& =1-\frac{4 g}{(1+g)^{2}} \cos ^{2}(\mu / 2) .
\end{aligned}
$$

This is illustrated in Figure 2, which shows the surface generated by Eq.(3). In the limit $g=1$ we obtain the usual relation,

$$
\frac{l}{2 f}=\sin (\mu / 2) \text {. }
$$

From the first line of Eq.(3), we see that the oscillatory condition, $|\cos \mu| \leq 1$, requires the inequality,

$$
\frac{1-g}{1+g} \leq \frac{l}{2 f} \leq 1,
$$

which is also evident in Figure 2.

\section{$3 \beta$, FROM THE TRANSFER MATRIX}

In CR, the eigenvectors of $M$ are related to the "lattice functions" of the cell. In the $\overline{\overline{\text { limit }}} g=1$, we are familiar

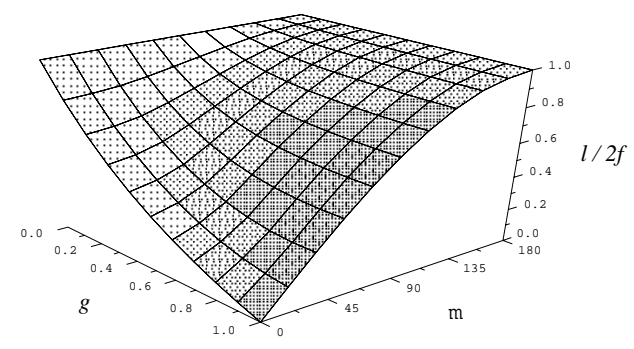

Figure 2: Ratio of quarter-cell length to focal length.

with the relationship,

$$
\frac{1}{\mu} \ln \underline{\underline{M}}_{\mathrm{CR}}=\left(\begin{array}{cc}
\alpha & \beta \\
-\gamma & -\alpha
\end{array}\right) .
$$

This representation is possible because of the symplecticity of $\underline{\underline{M}}$ CR. Although the $\underline{\underline{M}}$ of Eq.(1) is not symplectic, we can generalize Eq.(5) to it by factoring out the adiabatic damping. That is, $e^{\Gamma} \underline{\underline{M}}$ is symplectic, so that we can write

$$
\frac{1}{\mu} \ln \left(e^{\Gamma} \underline{\underline{M}}\right)=\frac{1}{\mu}(\Gamma+\ln \underline{\underline{M}})=\left(\begin{array}{cc}
\alpha & \beta \\
-\gamma & -\alpha
\end{array}\right)
$$

in place of Eq.(5). ${ }^{1}$ Because of Eq.(2) and Eq.(1), we can obtain the same result by rescaling $\underline{\underline{O}}$, thereby making it symplectic:

$$
\underline{\underline{O}}_{\text {symp }} \equiv g^{-1 / 2} \underline{\underline{O}}=\left(\begin{array}{cc}
g^{-1 / 2} & l\left(g^{-1 / 2}+g^{1 / 2}\right) / 2 \\
0 & g^{1 / 2}
\end{array}\right)
$$

Effectively, this removes the "damping" due to acceleration.

With this trick, we can calulate a "beta function" from the off-diagonal component of $\underline{\underline{M}}$ in the usual circular reasoning manner.

$$
\beta \sin \mu=e^{\Gamma} M_{12}=\frac{1}{g} M_{12}
$$

Using Equations (1) and (3) and rearranging terms a little provide the result.

$$
\beta / l=\frac{2+g+1 / g}{2 \sin \mu}\left(1+\sqrt{1-\frac{4 g}{(1+g)^{2}} \cos ^{2}(\mu / 2)}\right)
$$

\footnotetext{
${ }^{1}$ Notice that $e^{\Gamma} \underline{\underline{M}}, \underline{\underline{M}}$, and $\ln \underline{\underline{M}}$ all possess the same eigenvectors.
} 
This is $\beta$ at the location of the focussing quad; at the defocussing quad, merely replace $1+\sqrt{\cdots}$ with $1-\sqrt{\cdots}$. A family of these curves is plotted in Figure 3 for discrete values of $g$ between 0.1 and 1 . Unrealistically extreme accel-

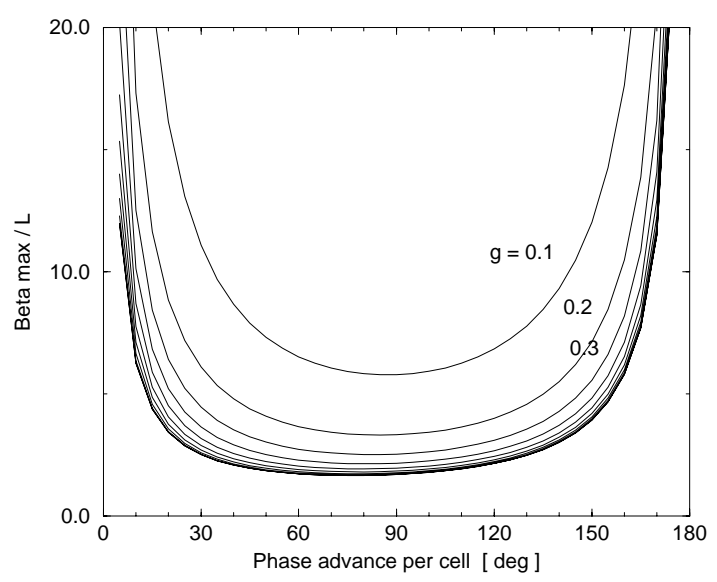

Figure 3: Maximum beta in the cell.

eration would be required to observe a significant deviation from the $g=1$ limit.

\section{PROPagating LatTiCE FUNCTIONS}

In passing, let us compare expressions for "propagating" lattice functions in both CR and ST. In ST one defines "lattice functions" in terms of the covariance of the beam.

$$
\underline{\underline{C}}=\left(\begin{array}{cc}
\left\langle x^{2}\right\rangle & \left\langle x x^{\prime}\right\rangle \\
\left\langle x^{\prime} x\right\rangle & \left\langle x^{\prime 2}\right\rangle
\end{array}\right) \equiv \operatorname{det}(\underline{\underline{C}})^{1 / 2}\left(\begin{array}{cc}
\beta & -\alpha \\
-\alpha & \gamma
\end{array}\right)
$$

This is in agreement with Eq.(5) when the covariance matrix is that of an invariant ellipse of the one-turn matrix, $\underline{\underline{M}}_{\mathrm{CR}}$. However, the beam need not be matched to the machine, so that $\alpha$ and $\beta$ are arbitrary here. Let $\underline{\underline{M}}_{B A}$ symbolize the linear transfer matrix from point $\mathrm{A}$ to point $\mathrm{B}$ in the machine, so that

$$
\underline{\underline{C}}_{(B)}=\underline{\underline{M}}_{B A} \cdot \underline{\underline{C}}_{(A)} \cdot \underline{\underline{M}}_{B A}^{T} .
$$

Combining this with the definition in Eq.(7) provides the following. To save space, let $D \equiv \operatorname{det}\left(\underline{\underline{M}}_{B A}\right)$.

$$
\left(\begin{array}{cc}
\beta & -\alpha \\
-\alpha & \gamma
\end{array}\right)_{(B)}=D^{-1} \underline{\underline{M}}_{B A}\left(\begin{array}{cc}
\beta & -\alpha \\
-\alpha & \gamma
\end{array}\right)_{(A)} \underline{\underline{M}}_{B A}^{T}
$$

On the other hand, because of the connection with the period-advance map via Eq.(5),

$$
\left(\begin{array}{cc}
\alpha & \beta \\
-\gamma & -\alpha
\end{array}\right)_{(B)}=\underline{\underline{M}}_{B A} \cdot\left(\begin{array}{cc}
\alpha & \beta \\
-\gamma & -\alpha
\end{array}\right)_{(A)} \cdot \underline{\underline{M}}_{B A}^{-1} .
$$

This is valid even for $g<1$ : according to Eq.(6), we simply subtract $\Gamma / \mu$ from both sides of the equation.

Superficially Eq.(8) and Eq.(9) look different, but it is a trivial exercise to show that they are, in fact, equivalent.

\section{ERROR IN PHASE ADVANCE}

The accelerating FODO cell that we have described, while enabling us to calculate exact quantities, is not actually used as the basic unit of "periodically" structured linacs. In extending CR to realistic linacs, one approach is to ignore acceleration except for its effect on scaling the transverse phase space coordinates. While $\beta$ depends on $g$, which changes from cell to cell, this can be safely ignored because the dependence is so weak for reasonable values of $\Delta E / E$. The phase advance per cell, $\mu$, also changes very little, but, unlike $\beta$, phase accumulates as one progresses down a chain of cells. The error that one would make by neglecting its dependence on $g$ can become significant after a sufficient number of cells. An estimate of this effect is shown in Figure 4. For a given $g$ and $\mu$, Eq.(3) is first

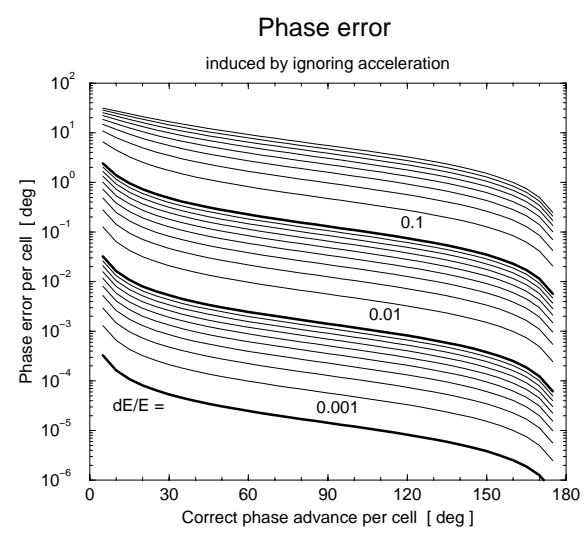

Wed Nov 10 13:10:43 1999

Figure 4: Phase error per cell induced by ignoring acceleration.

used to find the parameter $l / 2 f$. Using this value, Eq.(4) then provides the (incorrect) phase advance, say $\mu_{1}$, obtained by ignoring acceleration. The difference, $\mu-\mu_{1}$, is plotted in Figure 4 as a function of $\mu$, the correct phase advance, while labelling each curve by the corresponding $\Delta E / E=1 / g-1$. They indicate that, for a $90^{\circ}$ phase advance per cell, and for $\Delta E / E$ even as large as 0.02 , one would have to go through something like 100 cells before accumulating a $1^{\circ}$ phase error. This gives us an estimate of the extent over which we can trust standard CR formulae that involve the phase advance, provided we modify them to take phase space rescaling into account. 